\title{
Reducing Uncertainty in Shunt Damping by Model-Predictive Product Stiffness Control in a Single Point Incremental Forming Process
}

\author{
Florian Hoppe ${ }^{1, \mathrm{a} *}$, Maximilian Knoll ${ }^{1, \mathrm{~b}}$, Benedict Goetz ${ }^{2, \mathrm{c}}$, \\ Maximilian Schaeffner ${ }^{2, \mathrm{~d}}$ and Peter Groche ${ }^{1, \mathrm{e}}$
}

\author{
${ }^{1}$ Technische Universität Darmstadt, Institute for Production Engineering and Forming Machines, \\ 64287 Darmstadt, Germany
}

${ }^{2}$ Technische Universität Darmstadt, System Reliability, Adaptive Structures, and Machine Acoustics SAM, 64289 Darmstadt, Germany

a*hoppe@ptu.tu-darmstadt.de, bknoll@ptu.tu-darmstadt.de, 'cgoetz@sam.tu-darmstadt.de,

dschaeffner@sam.tu-darmstadt.de, 'egroche@ptu.tu-darmstadt.de

Keywords: forming, closed-loop control, prediction, shunt damping

\begin{abstract}
The stiffness of metal formed products strongly affects the dynamic behavior of structures in which they are integrated. Forming processes underlie short and long-term variations which cause the stiffness to be uncertain. In the application of resonant shunted piezoelectric transducers for vibration attenuation, uncertain stiffness may cause significant reduction in the vibration attenuation performance due to imprecise tuning. In the past, large efforts were made to control one or more geometrical feature of products while weightier features that cause uncertainty have not been addressed.

In this paper, a single point incremental forming process of a membrane-like spring element on a servo press with a 3 degrees of freedom drive system is investigated. This spring element is used in a beam support for lateral vibration attenuation with resonant shunted transducers as well as axial buckling stabilization.

To reduce uncertainty caused by process variations, an offline closed-loop control of product stiffness is presented. Different product and forming criteria are integrated into a control approach based on an optimization routine. By making use of a model-based prediction of the product properties, the approach shows how to realize a multi-objective control.
\end{abstract}

\section{Introduction}

In order to control the uncertainty of individual products along the product life cycle phases, not only controlled forming processes are required, but also a deeper understanding of the causes of uncertainty. This requires a holistic view of the production process, the product and the propagation of uncertainty.

Manufacturing processes are struggling with uncertainty caused by varying process conditions (e. g. lubrication, press and tool state), environmental conditions (e. g. shop temperature) and most importantly the actual material properties of the semi-finished product. Studies have shown that the yield strength of steel products may vary by $\pm 20 \%$ [1] and even the yield strength of the same steel coil contains variations of $\pm 5 \%$ over coil length and width [2]. It becomes clear that controlling process and environmental conditions is not sufficient to control the final product properties.

Closed-loop control in forming technology addresses the problem of maintaining a constant product quality when facing disturbances and has been intensively investigated in the past decades with great success [3]. To realize a closed-loop quality control, two things are fundamental. First, the machine and the tool have to provide the necessary flexibility to adapt the manufacturing process and on the other hand, the product quality has to be measurable. Depending on how feasible an online quality control is, an online or an offline closed-loop control can be implemented. Even though measuring the quality online is highly desirable, its expenses are often greater than the possible benefit. Hence, batch variations are visible only after the products have been produced. A wide-spread alternative is to statistically correlate process variables with quality variables and control the process variables online. 
Therefore, online closed-loop control focuses on controlling process or machine variables like forces or paths [3], e. g. in deep-drawing processes [4, 5]. Nevertheless, this approach only works up to a certain extent, as the quantitative relationship between process and product variables changes continuously over time, e.g. due to varying lubrication or tool wear. Offline closed-loop control, on the other hand, allows to control the final product properties, but is limited to adaptions solely between each process step. Therefore, they lack the ability to compensate batch variations. However, this disadvantage can be overcome by forwarding production data obtained from upstream processes and by identifying and compensating disturbances. [6].

The overarching goal in controlling product properties is to ensure the product functionalities during its usage phase. So far, modern adaptive offline closed-loop manufacturing systems mostly address geometric parameters, e. g. in bending processes $[6,7,8]$. Depending on the product requirements, its strength might be one of the most important features but cannot be controlled by now. In shunt damping, the product stiffness is a fundamental design variable. In a previous work, a closed-loop control of the axial stiffness of spring elements has been shown [9], but neglecting forming criteria like tensile strength or elongation at break during control. Taking such criteria into account requires a completely different control design. A controller based on an optimization problem benefits from a general framework that can take into account constraints like product or forming criteria. Such an approach has an international recognition as model-predictive control (MPC). First mentioned as receding horizon control in the 1960s and gaining major interest in the 1980s for controlling multivariable constrained systems, model-predictive control has been applied to industrial problems with outstanding success. Implemented in a single point incremental sheet forming process (SPIF), MPC has been proven to reach a good geometrical accuracy [10]. In this work, we show how an offline MPC can be implemented in a SPIF process using a 3D Servo Press to control the product stiffness whilst complying with forming and design constraints.

The SPIF process in particular is used to manufacture membrane-like spring elements that are components of piezo-elastic supports used for resonant shunt damping [11] as well as active buckling control [12]. In both applications, a beam with circular cross-section with piezo-elastic supports at both ends is used to either attenuate lateral beam vibration with resonant shunt damping or increase the maximum bearable axial load by active buckling control. In this paper, the focus lies on the application of resonant shunt damping.

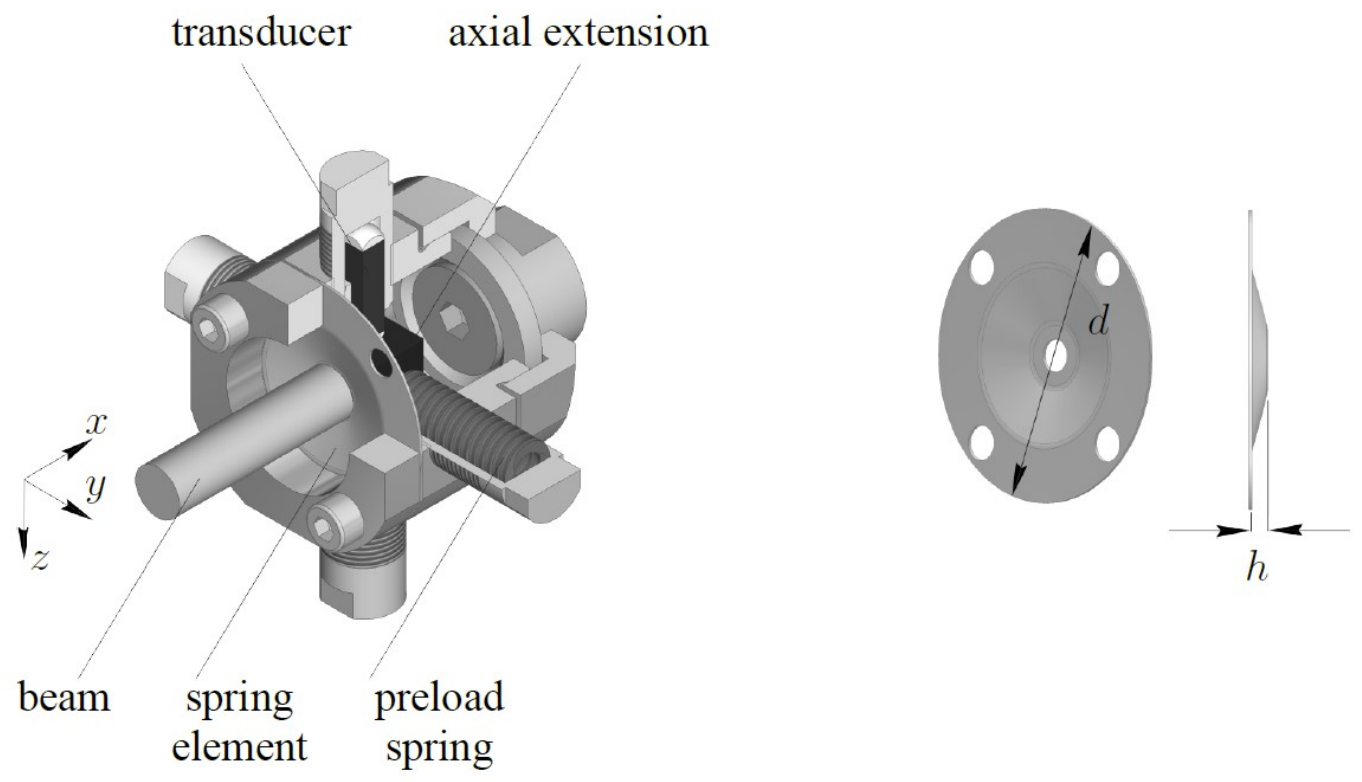

Fig. 1: CAD model of piezo-elastic support for vibration attenuation with shunted transducers (left) and CAD model of the spring element (right). 
Fig. 1 shows a sectional view of the piezo-elastic support. The central element of the support is an elastic membrane-like spring element that bears the axial and lateral loads and allows rotations in any plane perpendicular to the $x$-axis. Two piezoelectric stack transducers that are mechanically prestressed by allocated preload springs are connected to the beam via a relatively stiff axial extension. With this transducer arrangement, lateral beam-column deflections in $y$ - and $z$-direction are transformed into the transducer's axial deformation, and vice versa. By connecting the two piezoelectric transducers to resonant shunts, vibration attenuation of the first resonance mode in arbitrary lateral directions of the beam is possible.

The stiffness properties of the spring element are essential for the lateral dynamic vibration behavior of the beam. Ideally, the spring element has a high axial stiffness $k_{z}$ to bear the axial loads that act on the beam as well as a high lateral and low rotational stiffness to create a center of rotation at the beam's ends. This is achieved by the spring element shape shown in Fig. 1 with a conus created by the SPIF process. However, the high lateral and low rotational stiffness (not further investigated here) are in conflict to another, so that a compromise has to be found. For the usage of the spring elements, it is thus more important to have a precise lateral stiffness $k_{L}$ to get a reproducible system behavior.

If the stiffness properties are not met precisely, the lateral vibration behavior of the beam varies for different spring element products. Looking at the effect of fluctuating semi-finished product properties on lateral stiffness, it becomes clear that an uncontrolled forming process later leads to high fluctuations in vibration attenuation. Fig. 2 shows the effect of fluctuating elastic modulus and sheet thickness on the lateral stiffness $k_{L}$ of the spring element. Typical fluctuations in the production of semi-finished products already show a significant variance in product stiffness, which later influences the performance of vibration attenuation in the assembled state.
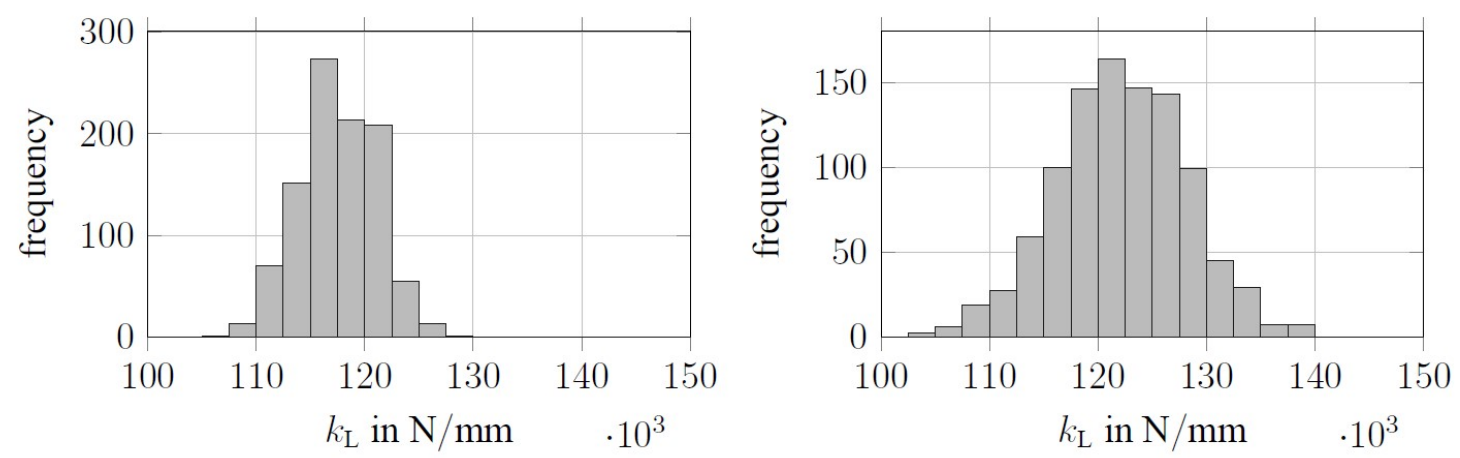

Fig. 2: Propagation of uncertainty of sheet metal properties on the lateral stiffness $k_{\mathrm{L}}$ using a monte carlo FEM simulation with $N=1000$ samples, (left) varying sheet thickness $t \sim \mathcal{N}\left(\mu_{t}=0.5 \mathrm{~mm}, \sigma_{t}=0.05 \mathrm{~mm}\right)$, (right) varying elastic modulus $E \sim \mathcal{N}\left(\mu_{E}=200 \mathrm{GPa}\right.$, $\left.\sigma_{E}=10 \mathrm{GPa}\right)$.

Fig. 3 shows the experimentally obtained vibration amplitude responses of a beam without shunts and with RL-shunts for two different spring elements with lateral stiffness $k_{\mathrm{L}_{1}}=67.93 \mathrm{~N} / \mu \mathrm{m}$ and $k_{\mathrm{L}_{2}}=79.59 \mathrm{~N} / \mu \mathrm{m}$. The resonance frequency of the beam without shunts differs by $3 \mathrm{~Hz}$. The resonant shunt is tuned to the resonance frequency of the beam with product 1 and achieves optimal vibration attenuation of the first resonance frequency. For the beam with product 2, thus, the shunt is mistuned and the vibration attenuation becomes suboptimal with the maximum vibration amplitude increased by $1.63 \mathrm{~m} / \mathrm{s}^{2}$. A precisely controlled lateral stiffness of the spring element is thus very desirable in the application of resonant shunt damping as it may reduce uncertainty in the vibration attenuation for varying products.

For the production of the membranes, a SPIF process shown in Fig. 4 has been set up. While a round blank is clamped at its edge, a ball tip travels circularly over the sheet surface and forms a freely programmable spring element shape. Table 1 sums up the product specifications for the spring element 

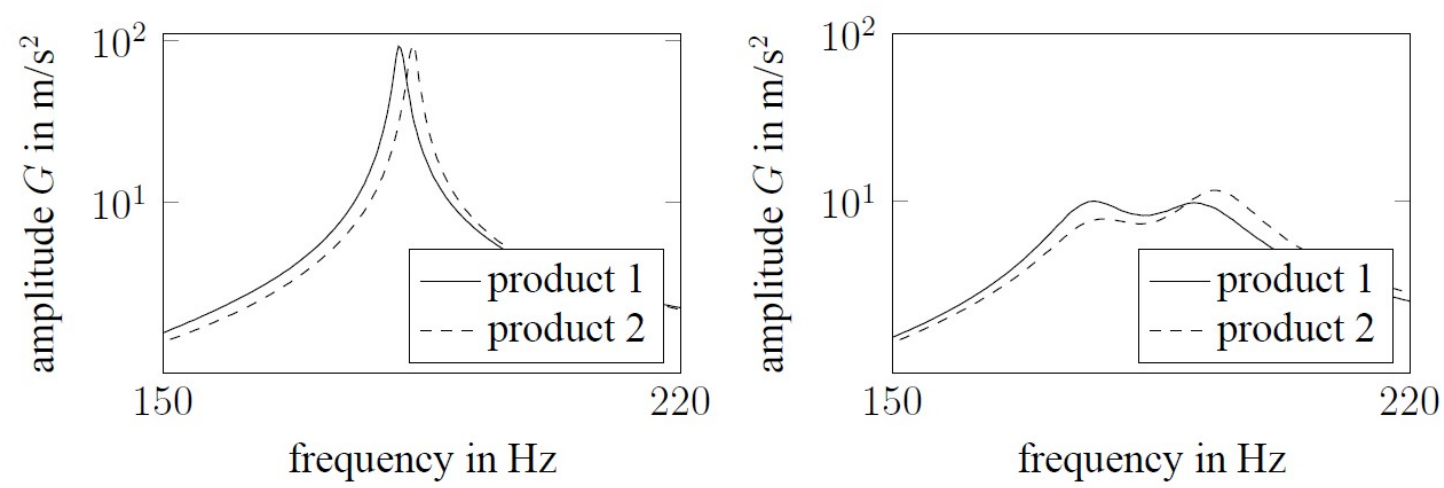

Fig. 3: Vibration amplitude response in the resonance frequency range without shunt (left) and vibration amplitude response with RL-shunt (right) for two membrane-like spring elements.
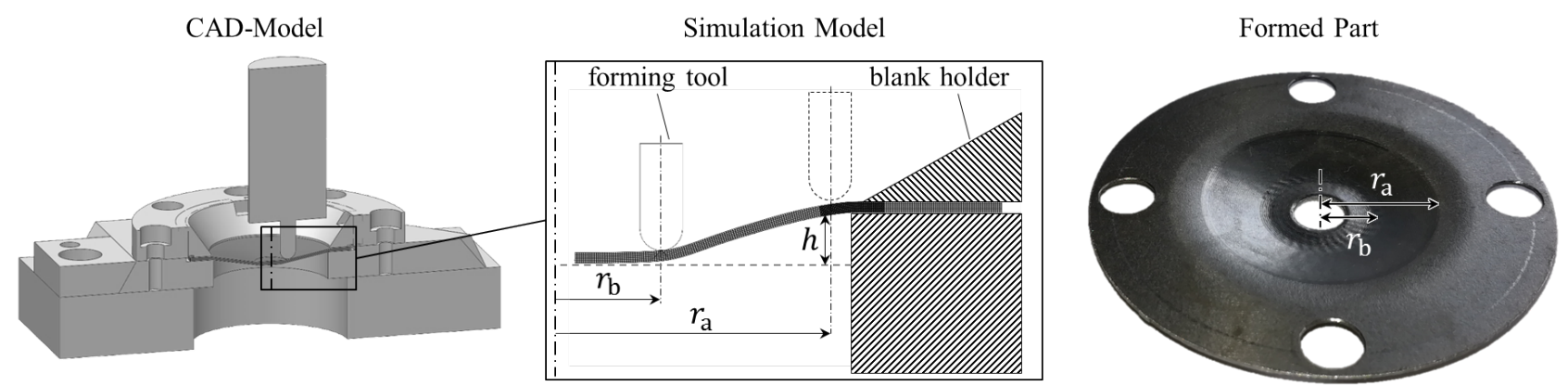

Fig. 4: Implemented Single-Point-Incremental Forming Process, (left) CAD Model of the tool, (middle) the abstraction to a 2D FE Simulation, (right) a formed spring element.

coming from the vibration attenuation design. While a specific lateral stiffness is required, buckling stabilization requires the axial stiffness to be maximized. In order to meet all specifications as well as possible, tolerances must be exploited. For this purpose, the tolerances for $r_{\mathrm{a}}$ and $r_{\mathrm{b}}$ offer room for the process to be adjusted without violating the geometry specifications.

Table 1: Specifications for the spring element

\begin{tabular}{l|l|l}
\multicolumn{2}{l|}{ Specification } & Target Value \\
\hline \multirow{3}{*}{ product } & lateral stiffness $k_{\mathrm{L}}$ & constant \\
& axial stiffness $k_{\mathrm{z}}$ & maximum \\
& height $h$ & $2.32 \mathrm{~mm}$ \\
& upper radius $r_{\mathrm{a}}$ & $10 \mathrm{~mm} \leq r_{\mathrm{a}} \leq 14 \mathrm{~mm}$ \\
& lower radius $r_{\mathrm{b}}$ & $4 \mathrm{~mm} \leq r_{\mathrm{b}} \leq 8 \mathrm{~mm}$ \\
\hline \multirow{2}{*}{ forming } & Maximum von Mises stress $\sigma_{\text {mis,max }}$ & $\leq$ tensile stress $R_{\mathrm{m}}$ \\
& logarithmic elongation $\varepsilon$ & $\leq$ elongation at break $\varepsilon_{\text {break }}$
\end{tabular}

\section{Method}

The challenge in controlling forming processes with multiple criteria is the lack of appropriate models. A novel approach is presented that takes into account a desired product stiffness of spring cups as well as forming criteria using the concept of model-predictive control. Three functions are required for this:

1. Model Modeling the forming process and the subsequent load of the product allows to determine the final product stiffness beforehand. 
2. Optimization Based on the model output, an objective function can be defined that the optimizer will try to minimize. In terms of a closed-loop control, the minimization of a quadratic error between desired and actual values is a common practice. Furthermore, constraints can be set to limit the optimizer to technically useful solutions.

3. Feedback As the optimizer is based on a model with limited accuracy and which is always affected by uncertainty, feeding back the measured control variable is inevitable for closed-loop control.

Fig. 5 shows how to integrate these functions into in a closed-loop forming process. At first, the optimizer iterates the regulating variables $\boldsymbol{u}_{\text {mdl }}$ until a desired output $\boldsymbol{k}_{\text {des }}$ is reached. The optimal values $\boldsymbol{u}_{\mathrm{opt}}$ are then passed to the machine that formes the spring element. As the model used cannot predict all kinds of disturbances that occur during the forming operation, the measured values $\boldsymbol{k}_{\text {meas }}$ are fed back to the model which will consider its error for the next part. As this approach does not allow for excessive computations, fast but precise models are required. Pure analytical models are fast but require a sufficient precision, they are only feasible for some forming processes, e. g. bending, but not processes like deep-drawing or SPIF. Numerical models offer a high degree of approximation and resolution in time and space but have not been used for controlling forming processes [3]. Therefore, the model used in this work includes a 2D FEM of the forming process that calculates the expected stresses, elongations and resulting geometry. Based on the geometry, a computationally efficient linear 3D FE model simulates the displacement of the product under load in axial and lateral direction and determines the corresponding stiffness. As this model approach is based on a physical approach, any variation of geometry or material properties, e. g. sheet thickness or elastic modulus can be taken into account. This is especially helpful if batch variations can be measured or estimated beforehand such that the model of the closed-loop control can be adapted immediately.

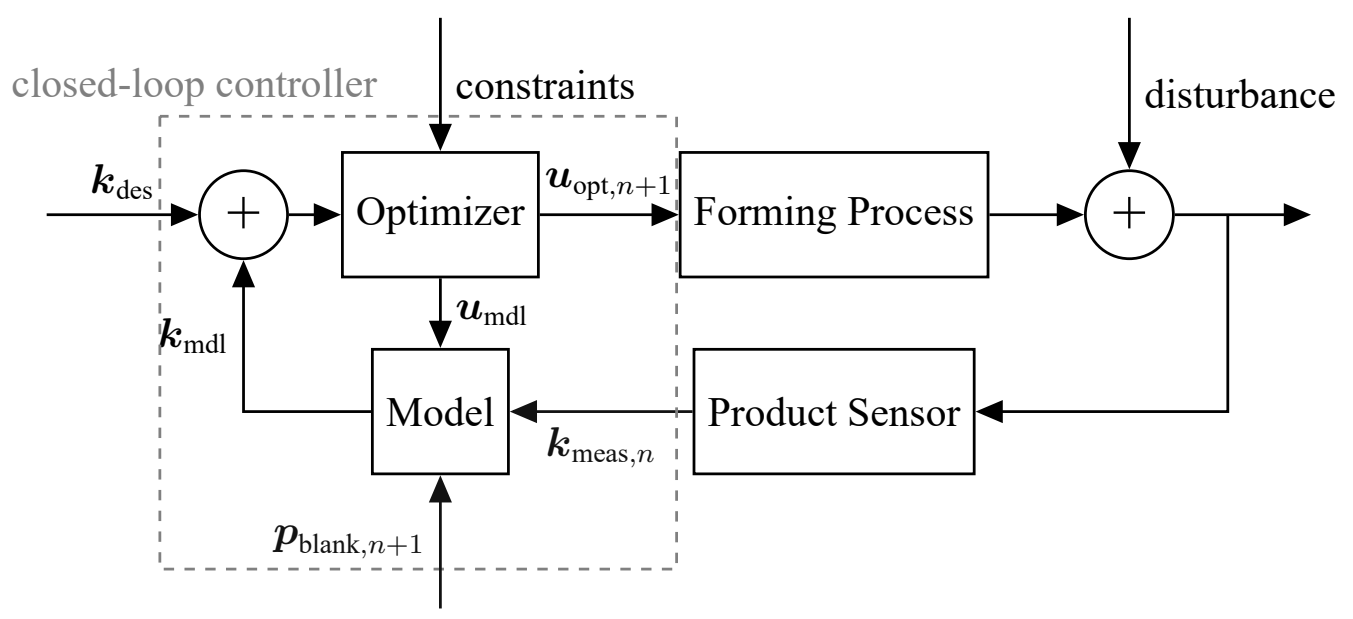

Fig. 5: Closed-loop control of the forming process designed as a model-predictive control

\section{Modeling}

The objective of the model is to allow for optimizing the control variables $\boldsymbol{u}_{\mathrm{mdl}}=\left[r_{a}, r_{b}\right]^{\mathrm{T}}$ to fit the desired product stiffness. As the formability is a crucial criterion, the optimization should take the occuring von Mises stresses and logarithmic elongations into account.

As outlined in [13], mathematical optimization of metal forming using an FEM consists of two major phases: modelling and solving of the optimization problem. While solving the problem can rely on a large optimization toolbox, the modelling part is a bottle neck. To perform an efficient optimization, a differentiable model is required. The Response Surface Methodology (RSM), first presented by Box and Wilson [14] has gained fame in the last decades for creating auxiliary models for optimization problems. Based on a variation of important process factors, a polynomial regression model 
is derived to fit the data. The polynomial aims to be of low-order and can be formulated in a general form $\boldsymbol{y}=\boldsymbol{\theta} \boldsymbol{x}+\boldsymbol{\xi}$. In this notation, $\boldsymbol{y}$ are the simulation response (geometry, von Mises stresses, elongations), $\boldsymbol{x}=\left[r_{\mathrm{a}}, r_{\mathrm{b}}\right]^{\mathrm{T}}$ are the input variables, $\boldsymbol{\theta}$ and function parameters to be fit and $\boldsymbol{\xi}$ process noise (e.g., numerical and model uncertainty). Increasing the order of the polynomial will succeedingly improve the result by means of lower residua but will end in overfitting. Therefore, in RSM, the order of the model is only increased if the statistical tests are not satisfactory [15]. This benefits the model-predictive control which is well-investigated regarding feasibility, stability and performance for linear systems while in the non-linear area still a lot of obstacles need to be overcome [16].

A closed-loop control for the axial stiffness using an auxiliary model based on Abaqus FE simulations has been shown in [9]. In that study, a first-order polynomial model has been used for predicting the axial stiffness change, i. e. the gradient. The closed-loop control used that gradient to gradually reach a desired axial stiffness. Still, that approach only took the axial stiffness into account and ignored other parameters as well as boundary conditions. Therefore we focus on controlling the lateral stiffness while complying with the constraints. Such a multi-objective approach requires a model for each objective, including the constraints.

\section{Finite element modeling}

To determine the stiffness for the optimizer, the formed product geometry is estimated using a static general forming simulation in Abaqus. The three dimensional SPIF process is implemented as a two dimensional rotationally symmetrical process as shown in Fig. 4. The simulation is based on a Johnson cook material model for steel DC01 (1.0330) with parameters from the work of Landi et al.[17]. The sheet metal to be formed for the simulation consists of 1750 quad elements of the type CAX4R and was split in three sections. For the contact zones between rigid forming tool and workpiece it is assumed that by using a rolling ball as forming tool no friction occurs in and the friction for the two-dimensional forming simulation can be neglected.

Based on the 2D FE simulation, a reduced geometry model is derived using a multivariate regression model that fits the complete set of nodes $\boldsymbol{N}=\boldsymbol{f}\left(r_{\mathrm{a}}, r_{\mathrm{b}}\right)$. Using the assumption of symmetry, a $3 \mathrm{D}$ model is created, with which a linear elastic load is simulated. Fig. 6 shows the reduced geometry model coming from the FE forming simulation and the two load cases. With the help of a GOM Atos optical measuring system, formed spring elements were digitized and compared with the geometry of the reduced model. A high accuracy was observed, only the pillow effect in the middle of the element, which bases on the residual stresses, was underestimated by the simulation.

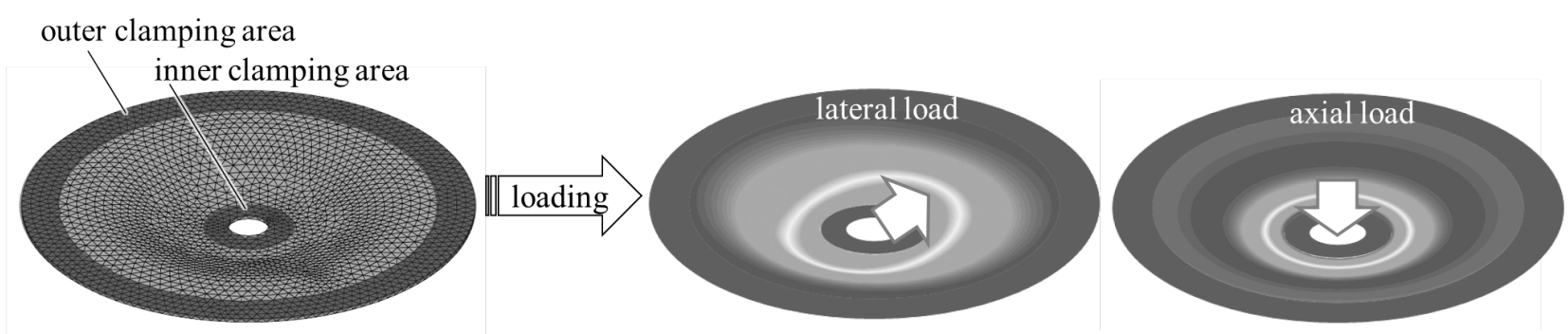

simulated geometry $\mid$ measured geometry

Fig. 6: Simulation of the stiffness using a geometry estimate from an FE forming simulation followed by a linear FE load simulation

Based on this model, a response surface can be generated for variable product features $r_{\mathrm{a}}, r_{\mathrm{b}}$. Fig. 7 shows the resulting surfaces for the lateral as well as the axial product stiffness. A strong correlation between $r_{\mathrm{a}}, r_{\mathrm{b}}$ and the stiffness can be observed which enables to control larger disturbances in the lateral stiffness of up to $20 \%$. Anyhow, also a correlation between $k_{\mathrm{L}}$ and $k_{\mathrm{z}}$ is visible which stands for a coupling of both properties. As a result, both, $k_{\mathrm{L}}$ and $k_{\mathrm{z}}$ are hardly controllable at the same time. The axial stiffness surface also appears smoother than the lateral stiffness. This has been lead back to the FE 
analysis which is more sensitive to the generated mesh in the lateral direction. The stochastic algorithm for generating the mesh therefore causes some stochastic variation in the $k_{\mathrm{L}}$. The influence of material parameters on the shape of the surface has also been investigated. While the elastic modulus has a linear influence and primary causes an offset, a sheet thickness of $-20 \%$ causes the lateral stiffness to slightly deviate in its gradient. As the gradient is decisive for the optimization and the closed-loop control, knowledge of the actual sheet thickness is expected to boost the controllers performance.
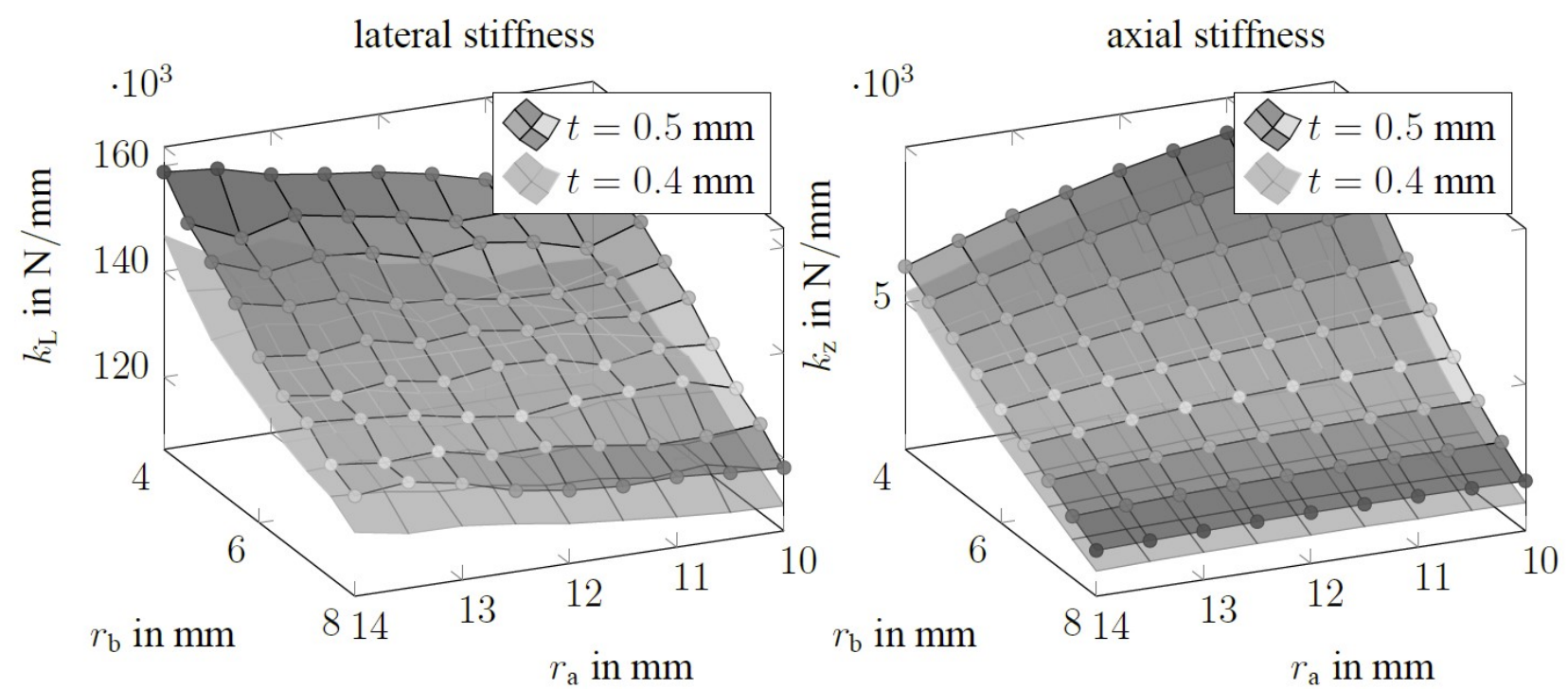

Fig. 7: Response surface of (left) lateral and (right) axial stiffnesses, obtained from an elastic FEM simulation

For optimizing the stiffness, a continuously differentiable function is required. Therefore, a 3rd order polynomial function of the form

$$
\begin{aligned}
k_{\mathrm{L}, i} & =\boldsymbol{\theta}_{\mathrm{L}}\left[r_{\mathrm{a}}, r_{\mathrm{b}}, r_{\mathrm{a}}^{2}, r_{\mathrm{a}} r_{\mathrm{b}}, r_{\mathrm{b}}^{2}, r_{\mathrm{a}}^{3}, r_{\mathrm{a}}^{2} r_{\mathrm{b}}, r_{\mathrm{a}} r_{\mathrm{b}}^{2}, r_{\mathrm{b}}^{3}, 1\right]_{i}^{\mathrm{T}} \\
k_{\mathrm{z}, i} & =\boldsymbol{\theta}_{\mathrm{z}}\left[r_{\mathrm{a}}, r_{\mathrm{b}}, r_{\mathrm{a}}^{2}, r_{\mathrm{a}} r_{\mathrm{b}}, r_{\mathrm{b}}^{2}, r_{\mathrm{a}}^{3}, r_{\mathrm{a}}^{2} r_{\mathrm{b}}, r_{\mathrm{a}} r_{\mathrm{b}}^{2}, r_{\mathrm{b}}^{3}, 1\right]_{i}^{\mathrm{T}}
\end{aligned}
$$

has been fit with the $i=1 . .81$ simulated lateral and axial stiffness. Fig. 8 exemplary shows the resulting polynomial fit for the lateral stiffness over $r_{\mathrm{b}}$ for $r_{\mathrm{a}}=10 \mathrm{~mm}$ and $r_{\mathrm{a}}=14 \mathrm{~mm}$.

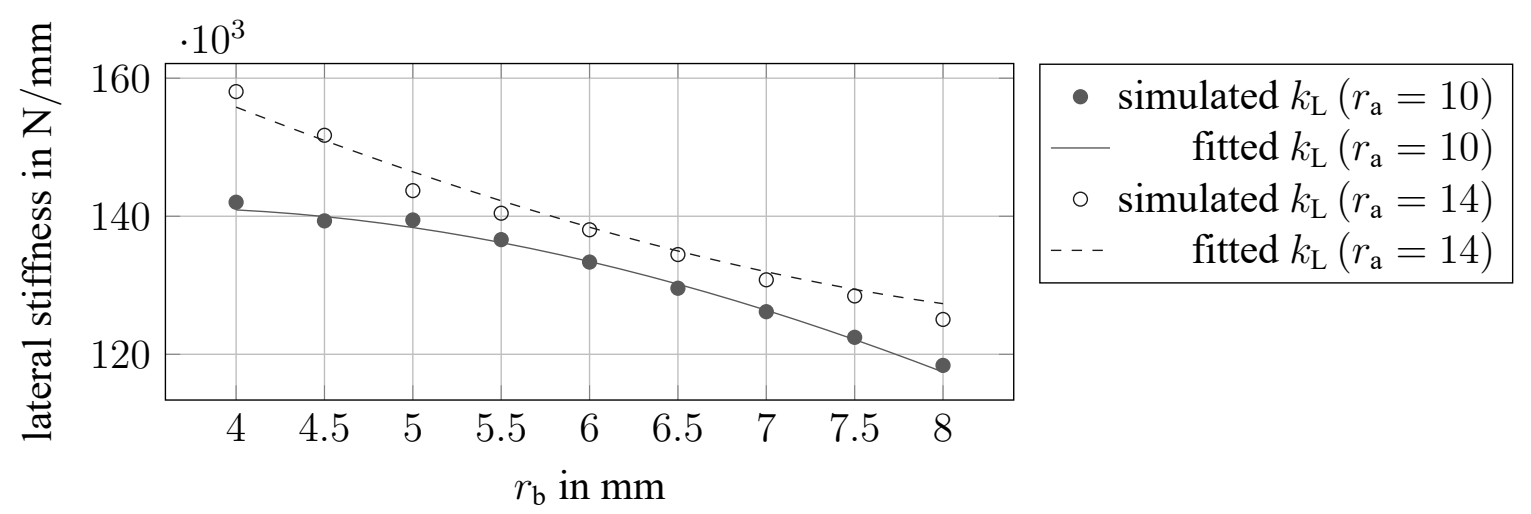

Fig. 8: Exemplary simulation response of the radial stiffness on the control variables $r_{\mathrm{a}}$ and $r_{\mathrm{b}}$ and the corresponding curve coming from the response surface 


\section{Forming Boundary Conditions}

While a closed-loop control may allow to process materials with uncertain properties, the same uncertainty affects the formability. For this purpose, the maximum stresses and elongations are extracted from the FE forming simulation and mapped just like the stiffness, shown in Fig. 9. Keeping these below the tensile stress $R_{\mathrm{m}}$ and elongation at break $\varepsilon_{\text {break }}$ is crucial for the forming process. If the actual $R_{\mathrm{m}}, \varepsilon_{\text {break }}$ are known, they can be forwarded to the MPC via the blank parameters $\boldsymbol{p}_{\text {blank }}$ such that the lateral stiffness can be controlled with respect to the actual formability.

Maximum logarithmic elongation

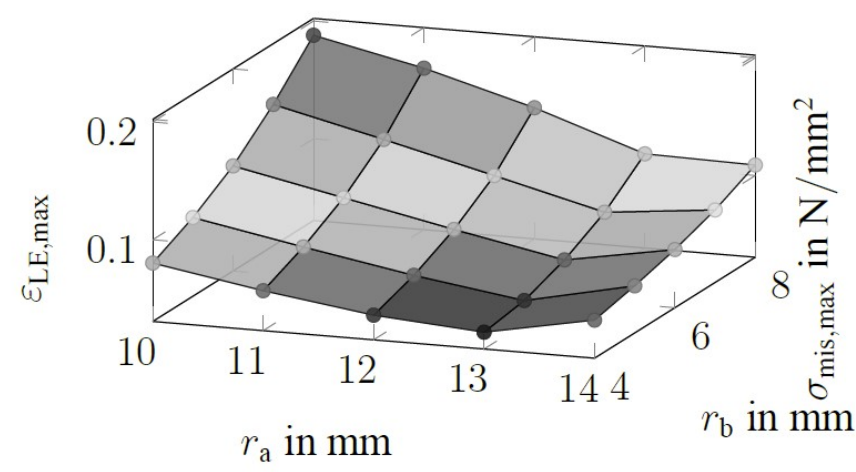

Maximum von Mises stress

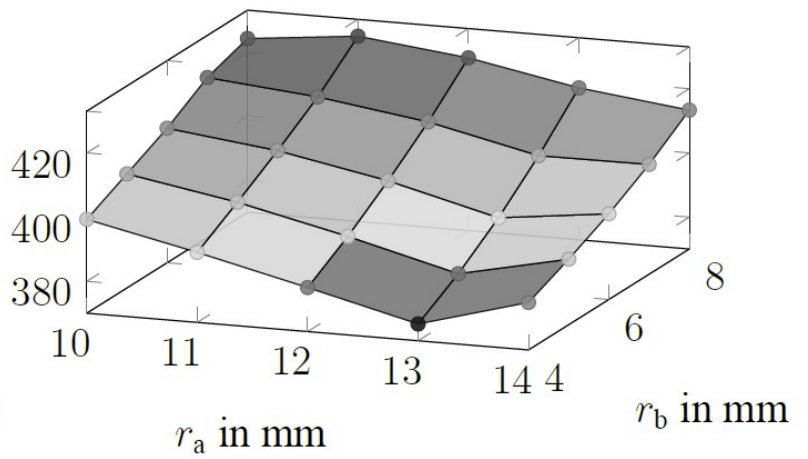

Fig. 9: Response surface of the forming properties (left) maximum logarithmic elongation and (right) maximum von Mises stress, occurring in the forming FEM simulation

\section{Optimization}

As depicted in Fig. 5, the closed-loop control can be formulated as an optimization problem that searches for the optimal parameters $\boldsymbol{u}=\left[r_{\mathrm{a}}, r_{\mathrm{b}}\right]^{\mathrm{T}}$ such that $k_{\mathrm{L}, \mathrm{mdl}}=k_{\mathrm{L}, \mathrm{des}}$ and $k_{\mathrm{z}}$ is maximal. By defining a quadratic error $\left(k_{\mathrm{L}, \mathrm{mdl}}-k_{\mathrm{L} \text {,des }}\right)^{2}$, an objective function can be set up to find the optimal parameters. In order to unite this criterion with maximizing $k_{z}$, weighing factors for each criterion are commonly introduced. Considering the constraints, the full optimization problem can be formulated as

$$
\begin{array}{cl}
\underset{\boldsymbol{u}}{\operatorname{minimize}} & J=q_{\mathrm{L}}\left(k_{\mathrm{L}, \mathrm{mdl}}-k_{\mathrm{L}, \mathrm{des}}\right)^{2}-q_{\mathrm{z}} k_{\mathrm{z}, \mathrm{mdl}} \\
\text { subject to } & \boldsymbol{u}_{\text {min }} \leq \boldsymbol{u} \leq \boldsymbol{u}_{\max } \\
& \varepsilon(\boldsymbol{u}) \leq \varepsilon_{\text {break }} \\
& \sigma_{\text {Mises }}(\boldsymbol{u}) \leq R_{\mathrm{m}}
\end{array}
$$

with the model-based estimation of the stiffness $k_{\mathrm{L}, \mathrm{mdl}}, k_{\mathrm{z}, \mathrm{mdl}}$, the desired lateral stiffness $k_{\mathrm{L} \text {,des }}$ and the weights $q_{\mathrm{L}}, q_{\mathrm{z}}$ weighing the importance of each stiffness.

As this approach is still open-loop it neglects uncertainty that causes the actual stiffness to deviate from the model-based stiffness prediction $k_{\mathrm{L}, \mathrm{mdl}}$. A closed-loop approach would require to minimize $\left(k_{\mathrm{L}, \mathrm{des}}-k_{\mathrm{L} \text {,meas, } \mathrm{n}}\right)^{2}$, by feeding back a measured lateral stiffness $k_{\mathrm{L} \text {,meas,n }}$ after each formed spring element $n$. Assuming an additive uncertainty $e_{n}$ in the model-based prediction at each step $n$ and that this error is approximately constant

$$
\begin{aligned}
k_{\mathrm{L}, \text { meas }, \mathrm{n}} & =k_{\mathrm{L}, \mathrm{mdl}}\left(\boldsymbol{u}_{\mathrm{opt}, n}\right)+e_{n} \\
e_{n+1} \approx e_{n} & =k_{\mathrm{L}, \text { meas }, \mathrm{n}}-k_{\mathrm{L}, \mathrm{mdl}}\left(\boldsymbol{u}_{\mathrm{opt}, n}\right)
\end{aligned}
$$


a closed-loop objective function

$$
\begin{aligned}
J_{n+1} & =q_{\mathrm{L}}\left(k_{\mathrm{L}, \mathrm{des}}-k_{\mathrm{L}, \text { meas }, \mathrm{n}+1}\right)^{2}-q_{\mathrm{z}} k_{\mathrm{z}, \mathrm{mdl}} \\
& =q_{\mathrm{L}}\left(k_{\mathrm{L}, \mathrm{des}}-\left(k_{\mathrm{L}, \mathrm{mdl}}\left(\boldsymbol{u}_{\mathrm{opt}, n+1}\right)+e_{n+1}\right)\right)^{2}-q_{\mathrm{z}} k_{\mathrm{z}, \mathrm{mdl}} \\
& =q_{\mathrm{L}}\left(k_{\mathrm{L}, \mathrm{des}}-\left(k_{\mathrm{L}, \mathrm{mdl}}\left(\boldsymbol{u}_{\mathrm{opt}, n+1}\right)+k_{\mathrm{L}, \text { meas }, \mathrm{n}}-k_{\mathrm{L}, \mathrm{mdl}}\left(\boldsymbol{u}_{\mathrm{opt}, n}\right)\right)^{2}-q_{\mathrm{z}} k_{\mathrm{z}, \mathrm{mdl}}\right.
\end{aligned}
$$

can be defined. After each forming step, new optimal regulating variables can be calculated by minimizing the objective function

$$
\boldsymbol{u}_{\mathrm{opt}, n+1}=\arg \min _{\boldsymbol{u}} J_{n+1}
$$

under the same constraints formulated in (4). Solving the optimization problems is performed with an interior-point method that realizes the constraints with logarithmic barrier function. At the beginning, the routine receives the differentiations of the objective function $\nabla_{\boldsymbol{u}} J_{n+1}(\boldsymbol{u}), \nabla_{\boldsymbol{u}}^{2} J_{n+1}(\boldsymbol{u})$ as well as the differentiations of the constraints. These can be computed on the basis of the models derived before $k_{\mathrm{L}}, k_{\mathrm{z}}, \varepsilon, \varsigma_{\text {Mises }}$. After each forming operation, the measured stiffness $k_{\mathrm{L}, \text { meas, } \mathrm{n}}$ is fed back into the routine causing an adaption of $\boldsymbol{u}_{\mathrm{opt}, n+1}$ for the next operation.

Due to springback, the formed height is being controlled in a parallel closed-loop routine. Since the influence of $r_{\mathrm{a}}, r_{\mathrm{b}}$ on the height $h$ is negligible, it will not be considered in this formulation.

\section{Results}

For the simulative analysis, batch fluctuations in the modulus of elasticity (190 - 200 GPa) were assumed. As the weighing parameters $q_{\mathrm{L}}, q_{\mathrm{z}}$ can be set individually, two different weights have been compared. A desired lateral stiffness $k_{\mathrm{L} \text {,des }}=131 \mathrm{~N} / \mu \mathrm{m}$ has been set which the controller tries to achieve. In contrast to the model for the optimizer which expects $E=200 \mathrm{GPa}$, the forming process is simulated with a varying elasticity and therefore feeds back a deviating stiffness. Fig. 10 shows the results of the closed-loop control in which the first 5 parts are produced with a batch of $190 \mathrm{GPa}$. After the first measurement, the controller reacts to the stiffness deviation and achieves the desired lateral stiffness. A new batch of $200 \mathrm{GPa}$ is then used. As this is the same elastic modulus that the model expects, model and actual stiffness are identical. Once the sixth part has been formed and measured, the controller notices that the deviation between model and measurement is $e_{6}=0$ and reacts again.
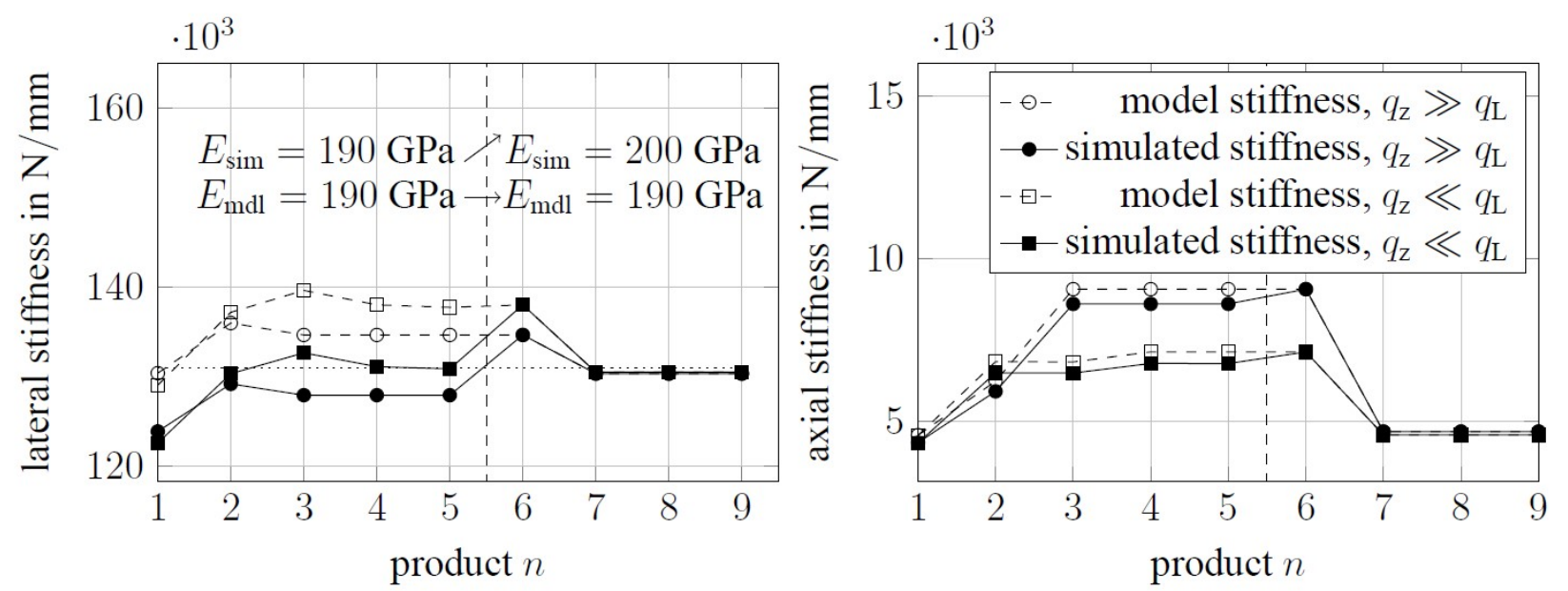

Fig. 10: Simulation of the closed-loop control with batch variations in elastic modulus of 190 to 200 GPa showing (left) the lateral stiffness being controlled, (right) the axial stiffness being maximized

A larger weighing of $q_{\mathrm{z}}$, will result in a less accurate $k_{\mathrm{L} \text {,meas }}$ to achieve a larger $k_{\mathrm{z}}$. In extreme case, if $q_{\mathrm{L}}=0$ is set to zero, the systems performs like an open loop. On the opposite side, a large $q_{\mathrm{L}}$ causes 
the controller to accurately follow $k_{\mathrm{L} \text {,des }}$ but finding a trade-off at the expense of $k_{\mathrm{z}}$. The controller then tries to stay on the same level line of $k_{\mathrm{L}}$ and get to the point on the line where $k_{\mathrm{z}}$ becomes maximum.

The presented method can be transferred to controlling both, axial and lateral stiffness or other properties. A major limitation is the coupling of the desired properties, i. e. the axial stiffness cannot freely be manipulated without affecting the lateral stiffness. Fig. 11 shows an example of controlling both stiffness values at the same time.

\section{Experimental Results}

To test the method under real and accurate conditions, a stiffness measurement has been set up using a mechanical strain testing machine. After each part formed, its lateral stiffness is determined as described in [18] and fed back to the closed-loop control which has been implemented in Matlab 2017a on the press control computer.

As the simulation revealed, uncertain properties of the material or semi-finished part largely influence the outcome of the forming process. By gaining knowledge about these properties and forwarding these information to the controller, disturbances can be compensated before the actual forming operation [6]. Therefore, an adaptive controller has been implemented in addition, by forwarding actual properties like sheet thickness $t$, elastic modulus $E$, poisson ratio $\nu$, tensile strength $R_{\mathrm{m}}$ and elongation at break $\varepsilon_{\text {break }}$ via the properties vector $\boldsymbol{p}_{\text {blank }}$ to the model (see Fig. 5).

To compare the adaptive and non-adaptive method, an open-loop steady-state with a sheet thickness of $t=0.4 \mathrm{~mm}$ has been established at first. Then, the sheet thickness is increased to $t=0.5$ $\mathrm{mm}$ and the actual model error $e_{n=4}$ as well as the actual stiffness $k_{\mathrm{L}, \mathrm{meas}, 4}$ is given to both controllers. While the adaptive controller receives this information, the non-adaptive controller only detects this deviation in the subsequent stiffness measurement.
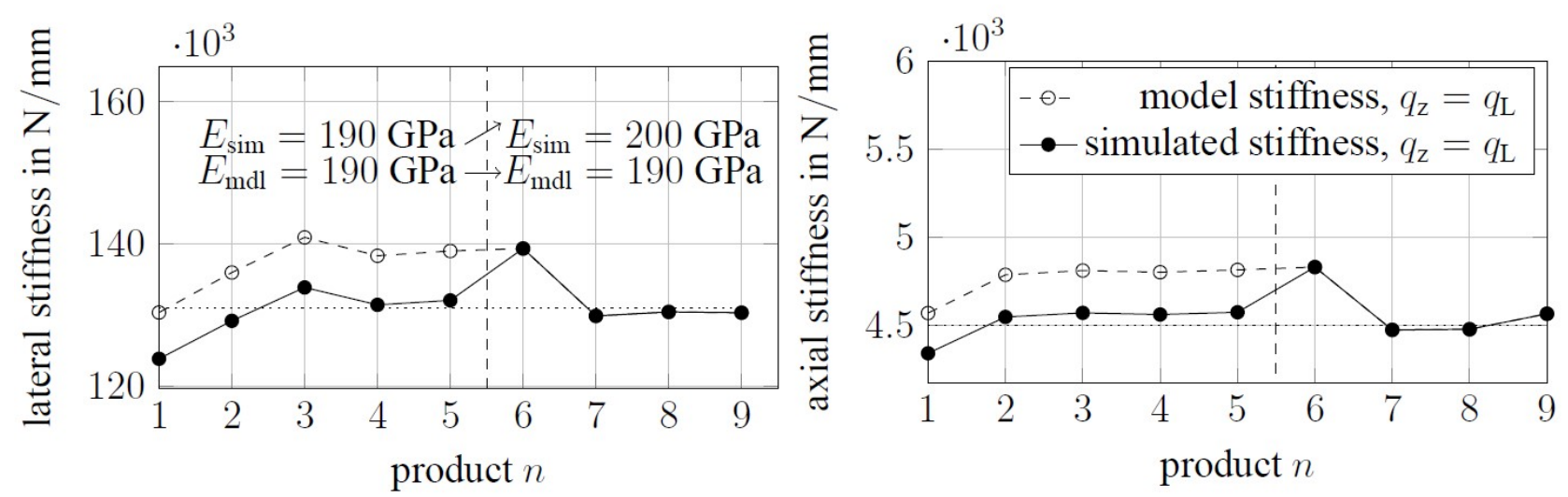

Fig. 11: Simulation of the closed-loop control with batch variations in elastic modulus of 190 to $200 \mathrm{GPa}$ showing (left) the lateral and (right) the axial stiffness stiffness being controlled.

Fig. 12 shows the experimental results for this setup. Even though the material properties of the first $n=4$ parts are identical, uncertainty in the sheet orientation (anisotropy), machine and clamping force cause a variation of $\pm 5 \mathrm{~N} / \mu \mathrm{m}$. In comparison to Fig. 7, a model error of $20 \mathrm{~N} / \mu \mathrm{m}$ can be determined. Then the controllers are given the negative control deviation $k_{\mathrm{L}, \mathrm{des}}-k_{\mathrm{L} \text {,meas, } 4}$ to produce the fifth part. As the error is negative, both controllers try to compensate it by increasing the stiffness. However, since the sheet thickness also increases, which generally leads to an increase in stiffness, the non-adaptive controller overshoots the target value. Surprisingly, the adaptive control manages to completely avoid the overshooting caused by the sheet thickness disturbance. This is only possible through the underlying physical model, with which the adaptive controller can adapt its model to new semi-finished product data. 


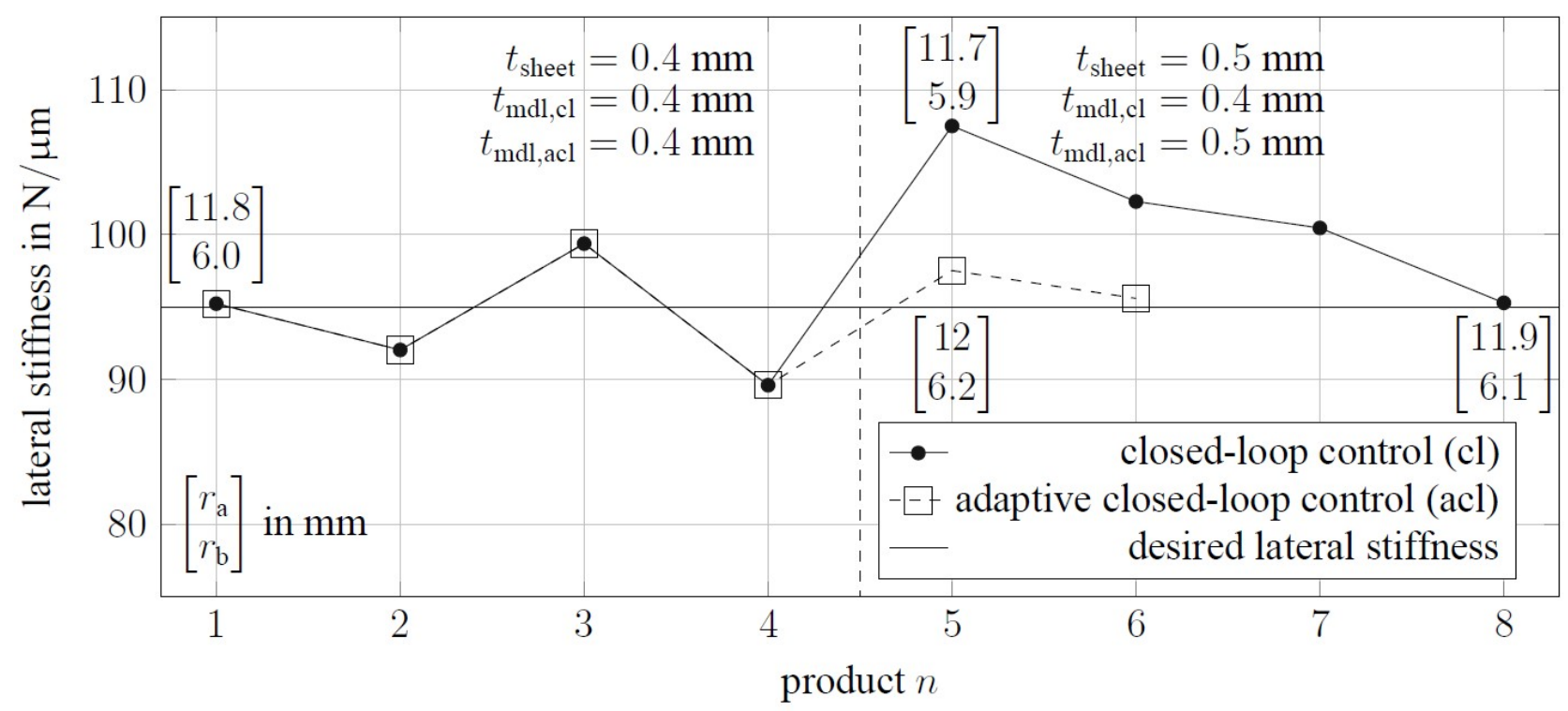

Fig. 12: Experimental results for the adaptive and non-adaptive closed-loop control with DC01 under a disturbance in sheet thickness of $0.1 \mathrm{~mm}$ after the 5 th product being formed. As the non-adaptive control cannot observe when and what causes the disturbance, it compensates the disturbance considerably slower than the adaptive control.

For the sixth component, the adaptive controller has already stabilized, while the non-adaptive controller still has to correct for the overshoot. Due to process fluctuations, the controller needs a few steps until it is back in steady state.

\section{Conclusion}

A model-based approach has been shown to control product properties in forming processes offline while complying with forming criteria. The presented method can be applied to a variety of forming processes to control. On the one hand, the prerequisites for this are that there are process parameters that can be adapted between each forming operation. On the other hand, deterministic models must be derived from simulations or experiments that map the influencing variables on product properties. The major advantage is that the optimization does not require explicitly invertible models. This may allow the use of empirical models created by machine learning, e.g. support vector regression, neural networks, gaussian process regression. The result is likely to be a highly non-convex optimization problem that requires a different solver. Nonetheless, the physical based model has shown to come with the benefit of allowing model adaptions when material deviations can be observed beforehand. An empirical model can also be used for adaptive control, but requires a large amount of data to learn the effect of all possible fluctuating parameters. Additive model errors can be compensated by the presented control, which is why quantitative deviations between model and measurement are insignificant. Therefore, the method shown can easily be applied to new processes and tools without a time-consuming learning phase. However, the method has so far been limited to offline control, since only the process state before and after the forming is taken into account. For an online control it is necessary to derive a differentiable model for every possible state during the forming process. This is currently still the subject of research. 


\section{Acknowledgements}

The results of this paper are achieved within the Collaborative Research Centre (SFB) 805 "Control of uncertainty in load-carrying mechanical systems" in subprojects "B2: Forming - Production families at equal quality", "C7: Structural Health Control of load-carrying mechanical systems" and "C2: Mechanical, mechatronic and adaptive technologies for the stabilization of beam-columns and truss structures" funded by the German Research Foundation (DFG). The authors wish to thank for funding and supporting the project.

\section{References}

[1] Dj.M. Maric, P.F. Meier and S.K. Estreicher: Mater. Sci. Forum Vol. 83-87 (1992), p. 119

[2] Jörg Heingärtner, Yasar Renkci, and Pavel Hora. "Application of non-destructive testing to control material properties of stainless steel in kitchen sink production". In: IDDRG 2013 Conference: Towards Zero Failure Production Methods by Advanced Modeling Techniques and a Process Integrated Virtual Control. Ed. by Pavel Hora. ETH Zurich, Institute of Virtual Manufacturing, 2013, p. 80. ISBN: 978-3-906031-34-7.

[3] C. Held, M. Liewald, and M. Sindel. "Untersuchungen zum Einfluss werkstofflicher Schwankungen innerhalb eines Coils auf die Umformbarkeit”. In: wt Werkstattstechnik online 99 (2009), pp. 732-739.

[4] Benny Endelt, Søren Tommerup, and Joachim Danckert. "A novel feedback control system Controlling the material flow in deep drawing using distributed blank-holder force". In: Journal of Materials Processing Technology 213.1 (2013), pp. 36-50. ISBN: 09240136. DOI: 10.1016/ j.jmatprotec.2012.08.003.

[5] Bernd Arno Behrens, J. W. Yun, and M. Milch. "Closed-Loop-Control of the Material Flow in the Deep Drawing Process". In: Advanced Materials Research 6-8 (2005), pp. 321-328. ISBN: 1662-8985. DOI: 10.4028/www.scientific.net/AMR.6-8.321.

[6] Peter Groche et al. "Blanking-bending process chain with disturbance feed-forward and closedloop control". In: Journal of Manufacturing Processes 24 (2016), pp. 62-70. ISBN: 15266125. DOI: $10.1016 /$ j.jmapro.2016.07.005.

[7] Jos Havinga and Ton van den Boogaard. "Estimating product-to-product variations in metal forming using force measurements". In: AIP Conference Proceedings. Author(s), 2017, p. 070002. DOI: $10.1063 / 1.5008077$.

[8] Jos Havinga et al. "Feedforward control of sheet bending based on force measurements". In: Journal of Manufacturing Processes 31 (2018), pp. 260-272. ISBN: 15266125. DOI: 10.1016/ j.jmapro.2017.10.011.

[9] Daniel Hesse, Florian Hoppe, and Peter Groche. "Controlling Product Stiffness by an Incremental Sheet Metal Forming Process". In: Procedia Manufacturing 10 (2017), pp. 276-285. ISBN: 23519789. DOI: 10.1016/j.promfg.2017.07.058.

[10] Haibo Lu et al. "Model predictive control of incremental sheet forming for geometric accuracy improvement". In: The International Journal of Advanced Manufacturing Technology 82.9 (2016), pp. 1781-1794. ISBN: 1433-3015. DOI: 10.1007/s00170-015-7431-5. DOI: https: //doi.org/10.1007/s00170-015-7431-5. 
[11] Benedict Götz, Roland Platz, and Tobias Melz. "Effect of static axial loads on the lateral vibration attenuation of a beam with piezo-elastic supports". In: Smart Materials and Structures 27.3 (2018), p. 035011. DOI: 10.1088/1361-665X/aaa937.

[12] Maximilian Schaeffner and Roland Platz. "Gain-scheduled H1 buckling control of a circular beam-column subject to time-varying axial loads". In: Smart Materials and Structures (2018). to appear. DOI: http://iopscience.iop.org/10.1088/1361-665X/aab63a.

[13] M.H.A. Bonte, A. H. van den Boogaard, and J. Huétink. "A Metamodel Based Optimisation Algorithm for Metal Forming Processes". In: Advanced methods in material forming. Ed. by D. Banabic. Berlin, Heidelberg: Springer-Verlag Berlin Heidelberg, 2007, pp. 55-72. ISBN: 978-3540-69845-6. DOI: 10.1007/3-540-69845-0_4.

[14] G.E.P. Box and K. P. Wilson. "K B. 1951. On the Experimental Attainment of Optimum Conditions". In: Journal of the Royal Statistical Society 13 (1951), pp. 1-45.

[15] Angela Dean, Daniel Voss, and Danel Draguljić, eds. Design and Analysis of Experiments. Second edition. Springer Texts in Statistics. Cham: Springer International Publishing, 2017. ISBN: 978-3-319-52250-0.

[16] Manfred Morari and Jay H. Lee. "Model predictive control: Past, present and future". In: Computers \& Chemical Engineering 23.4-5 (1999), pp. 667-682. ISBN: 0098-1354.

[17] L. Landi et al. "Sheets impact simulation for safety guards design: Experiments and correlation for FE Explicit models of non-alloy steel". In: Procedia Structural Integrity 8 (2018), pp. 3-13. ISBN: 24523216. DOI: 10.1016/j.prostr.2017.12.002.

[18] Benedict Götz et al. "Model verification and validation of a piezo-elastic support for passive and active structural control of beams with circular cross-section". In: Applied Mechanics and Materials, Trans Tech Publications 807 (2015), pp. 67-77. 